\title{
THE STOCHASTICITY OF MOTIONS IN THE TRIPLETS OF STARS AND GALAXIES
}

\author{
J.P. Anosova, L.G. Kiseleva, A.D. Chernin, A.V. Ivanov, \\ and V.V. Orlov
}

\begin{abstract}
The dynamical instability of motions is studied numerically for the triplets of stars and galaxies. The models of equal-mass components which are in rest initially, are investigated. The divergence of initially nearby trajectories close to exponential one is observed in the configurational as well as the phase space. The characteristic time of such divergence is less for the configurations which are initially hierarchical. The presence of significant dark matter distributed over the whole volume of system speeds up the rate of stochastisation considerably.
\end{abstract}

\section{INTRODUCT ION}

There is a tendency for stars and galaxies to group to clusters (Gorbatsky 1986, Batten 1975) of various multiplicity. Structurally, the most simple but in the same time nontrivial from the dynamical viewpoint are the triple stars and the triplets of galaxies. The catalogues of small groups of galaxies and multiple stars are the main source of observed information. The single homogeneous catalogue containing only the triplets of galaxies is the Karachentseva et al. catalogue $(1979,1987)$. Because there are no any effective analytical techniques for solving three-body problem,many authors use the numerical methods for study the dynamics of triplets (see, for instance, Valtonen 1988, A no sova 1986). In the most cases (90\%), as numerical experiments show, the triplets of stars are unstable with respect to disintegration on binary system and isolated body throwing out of the system. On the other hand, in the dynamics of triplets of galaxies we have 
the processes of merging which plays significant roles(Mamon 1987 , Alladin 1988). The result of these different processes is the same - the multiplicity of system changes. The purpose of this paper is to study of stochastic properties in the dynamics of triplets of stars and galaxies.

\section{MODELS AND METHODS}

In this paper the dynamical evolution of triplets of stars and galaxies with components of equal masses and zero initial velocities is investigated. Such initial conditions lead from cosmogonical considerations that the system forms from nonrotating quasistationary ga seous cloud by fragmentation. Moreover, the numerical simulations show the similarity of the dynamical evolution in plane and spatial cases. We used so called "softened" potential for galaxy-galaxy interactions (Aarseth,1963)

$$
\psi_{i j} \sim\left(\tau_{i j}^{2}+\varepsilon^{2}\right)^{-1 / 2}
$$

where $P_{i j}$ - is the distance between centers of galaxies, $\varepsilon-$ softening length which has the same order as half-mass radius of galaxy (Mamon,1987). To take into account the possibility of merging of galaxies at their close approaches we use the criterion of Roos and Norman (1979). For the systems of galaxies we also studied the case when the triplet is imbedded into dark matter which is distributed spherically over the whole volume by the law

$$
\rho(R) \sim\left\{\begin{array}{l}
\mathrm{R}^{-2}, \varepsilon \leq \mathrm{R} \leq \mathrm{R}_{\mathrm{h}} \\
0, \mathrm{R}>\mathrm{R}_{\mathrm{h}}
\end{array}\right.
$$

where $R$ - is the distance from the center of dark matter which coincides with the baricenter of triplet in starting moment. $R_{h^{-}}$is the limiting radius of dark matter distribution. The back action of galaxies to distribution of dark matter so as the dynamical friction considered is negligible. For the initial configurations used a configurational diagram $D$ (see Fig. 16 in Anosova 1986). In this case the initial conditions are determined by two coordinates $\xi$ and $\eta$ on area $D$. The dynamical systems of units have been used in the simulation where $m$ - is the average mass of component, $d$ - is the aver age size of system and $\tau$ - is the average crossing $\xi$ - time for the component of the system.

RESULTS

The effects of initial conditions variations.

The instability of results with respect to small variations of initial conditions is obtained in numerical simula- 
tions of the dynamics of triple systems Szebehely 1973,Agekian and Anosova 1977, A no sova and Zavalov 1988). In order to study a process of scattering of the nearby trajectories, we have pointed out the configurations inside the region $D$ by a step of $t$ ime $t=1$ of $2 \tau$ (see Figures $1-3$ ). The scattering of configurations of trajectories takes place - a "cloud" of point images becomes wider during a time and gradually fills the most part of the regiond $D:$ at $\left(\xi_{0}, n_{0}\right)=(0.22,0.45)$ during about $18 \mathrm{\tau}$; in the case of $\left(\xi_{0}, \eta_{0}\right)=(0.22,0.25)$ this time is equal to about 10 ; in the first case $\left(\xi_{0}, n_{0}\right)=(0.386,0.050)$ the imaging points fill a. strip 0.3 along the $\xi$ axis (during a time about $2 \tau$ ). A speed of scattering is different to these three points: it is faster for the hierarchial systems and cha in-like ones than for non-hierarchial systems with $\left(\xi_{0}, \eta_{0}\right)$ $=(0.22,0.45)$. In all three cases these estimates are smaller than a time of numerical instability caused by the errors of numerical integration ( $\sim 50 \tau$, see Skodrov et al. 1988). Let us follow a topological rebuilding of a family of configurations. The twisting and elongating of the segment takes place with a following self-crossing of imaging curve.

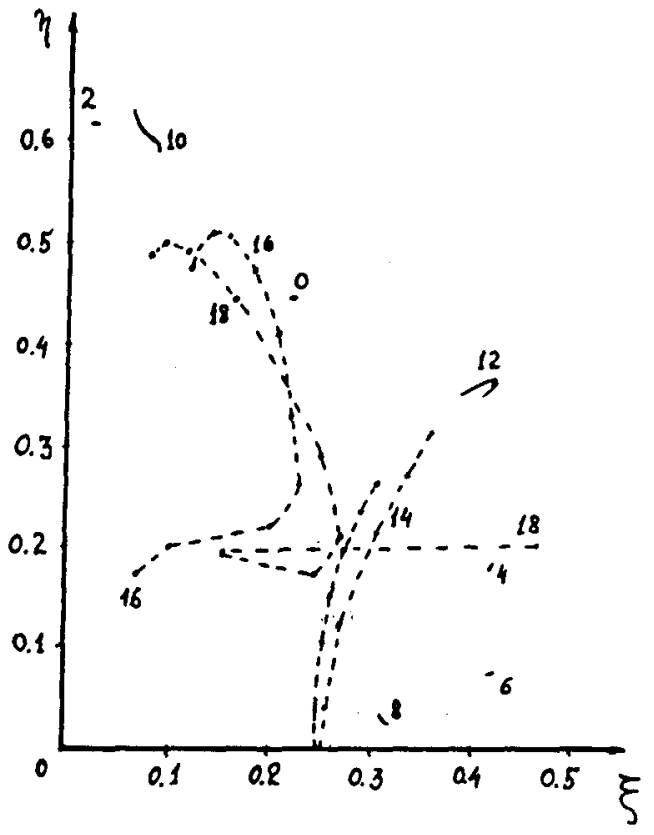

Figure $1:\left(\xi_{0}, n_{0}\right)=(0.22,0.45)$. 


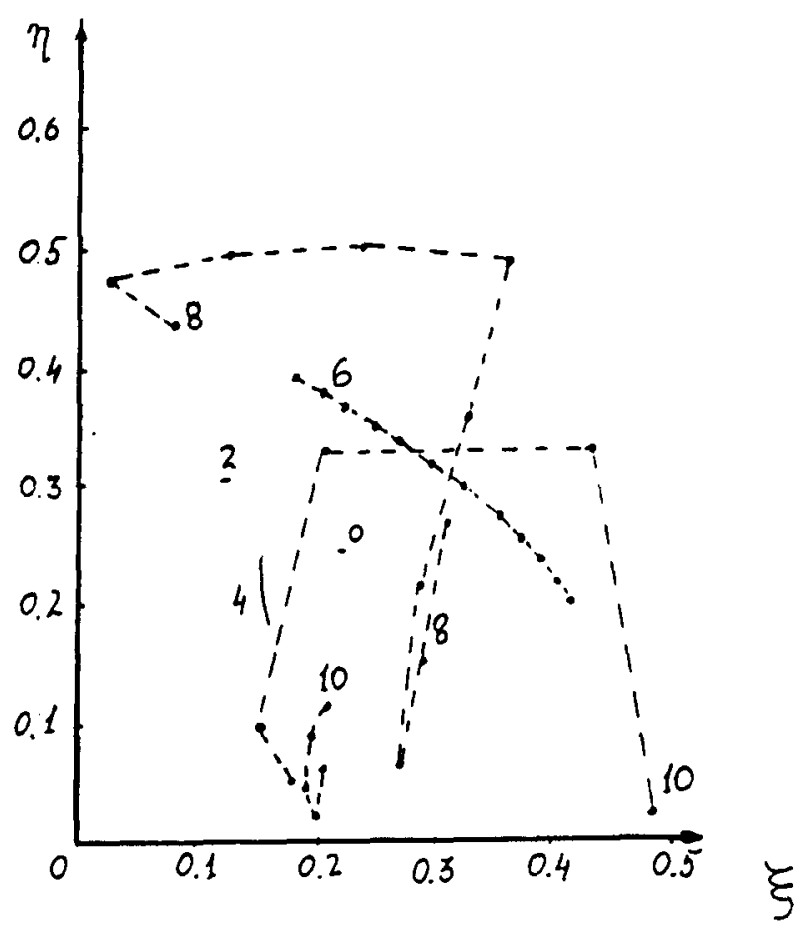

Figure $2\left(\xi_{0}, n_{0}\right)=(0.22,0.25)$.

The similar results for the ensemble of the model triplets of galaxies (with $\varepsilon=0.1 \mathrm{~d}$ ) are shown in Fig. 4. The 25 initial configurations were taken on the segment $\xi \in[0.22$, $0.23], n=0.45$. The evolution of this ensemble al so leads to reshaping and elongating of the segment. Finally, the imaging points fill the $D$ area accidentally with clear condensation near $\xi$ axis.

Quantitive characteristic of the dynamical chaos in the triplets:

Fast increasing of the distance between $t$ wo initially close trajectories in the configurational space directs to stochasticity of dynamics of triple systems. One can evaluate by using of the maximum Liapunov number (Lichtenberg, Lieberman 1982) the rate of divergence of initially nearby trajectories. We compute the maximum Liapunov number by $1 \mathrm{im}$

$$
\sigma(t)=t \rightarrow \infty, d(0) \rightarrow 0
$$

$1 \mathrm{im}(1 / t) \operatorname{nn}|\mathrm{d}(\mathrm{t})| /|\mathrm{d}(0)|$ using the method proposed Casartelli et al.(1976). It is impossible in practice to compute this number in the limit $t+\infty$, so the explicit behaviour of timedependent variable $\sigma_{1}(t)=\lim _{d(0) \rightarrow 0}(1 / t) \ln |d(t)| /|d(0)|$ was 


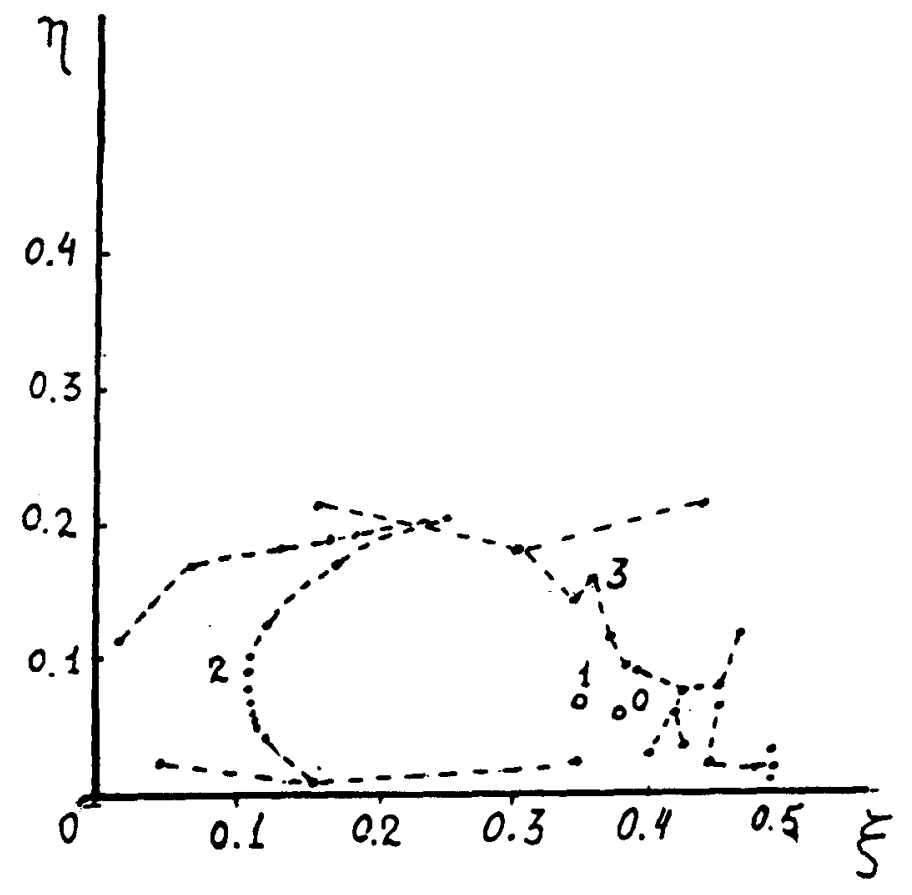

Figure 3: $\left(\xi_{0}, n_{0}\right)=(0.386,0.050)$.

studied. Figure 5 demonstrates ${ }_{1}(t)$ for several examples of evolution with and without dark matter in the system. The mass of the dark matter were taken in 10 times larger than the total mass of the triplet. As in the case of stars the whole systems were imbedded into distribution of dark matter. The computations show that $\sigma_{1}(t)>0$ in all examples, so we have the exponential divergence in average. Such behaviour is typical for dynamically unstable motions. For the models with distributed dark matter the maximum $L$ iapunov number is larger than in the case with isolated three-body system so the presence of significant hiding mass accelerates stochastisation.

\section{CONCLUSION}

The numerical simulation of the dynamic of triple systems of stars and galaxies shows that the timescale of stochastisation is considerably less than its lifetime. 

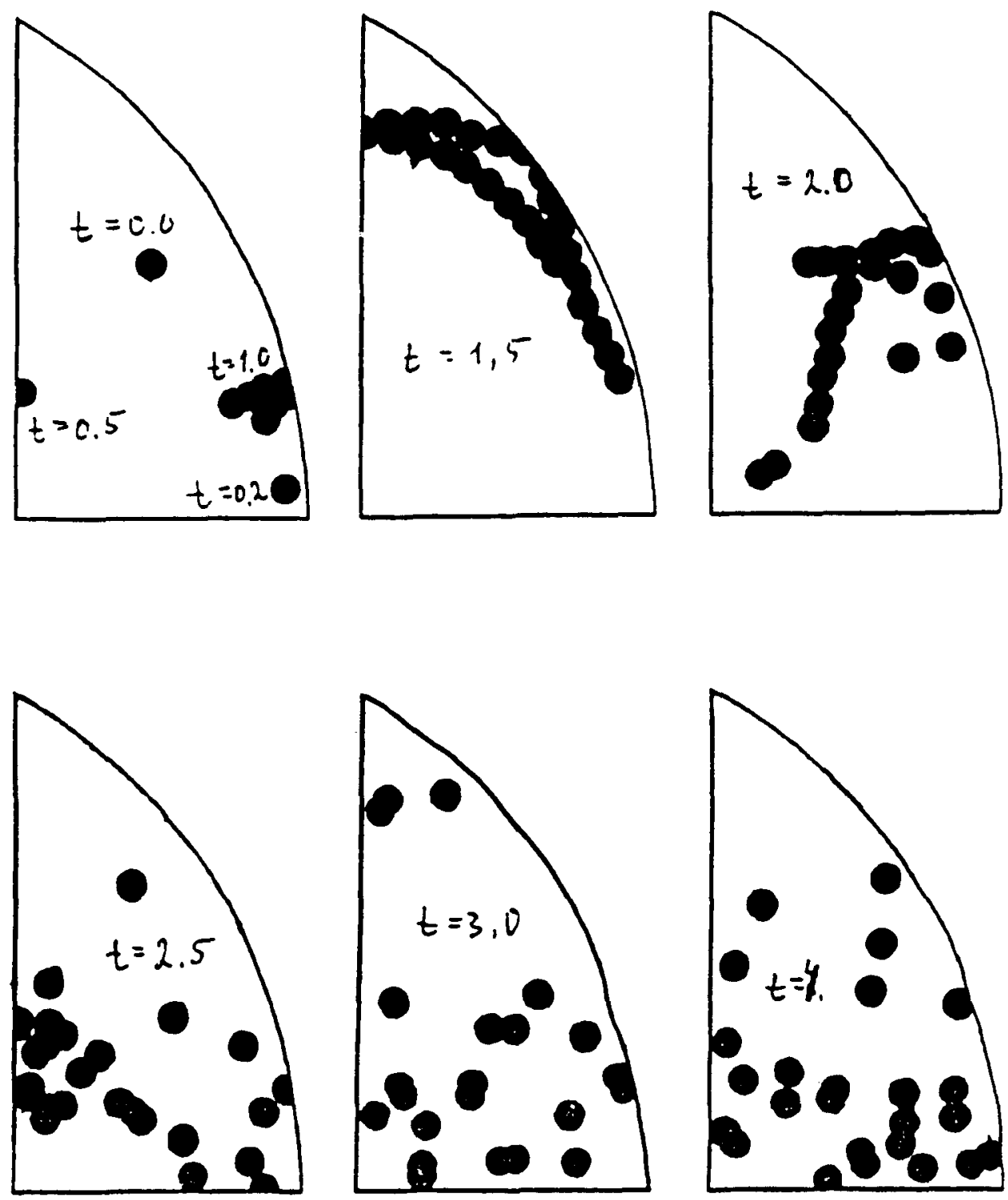

Figure 4: The disintegration of the ensemble of the triplets of galaxies, which lies initially on the segment $\xi \in[0.22,0.23], \eta=0.45$. 

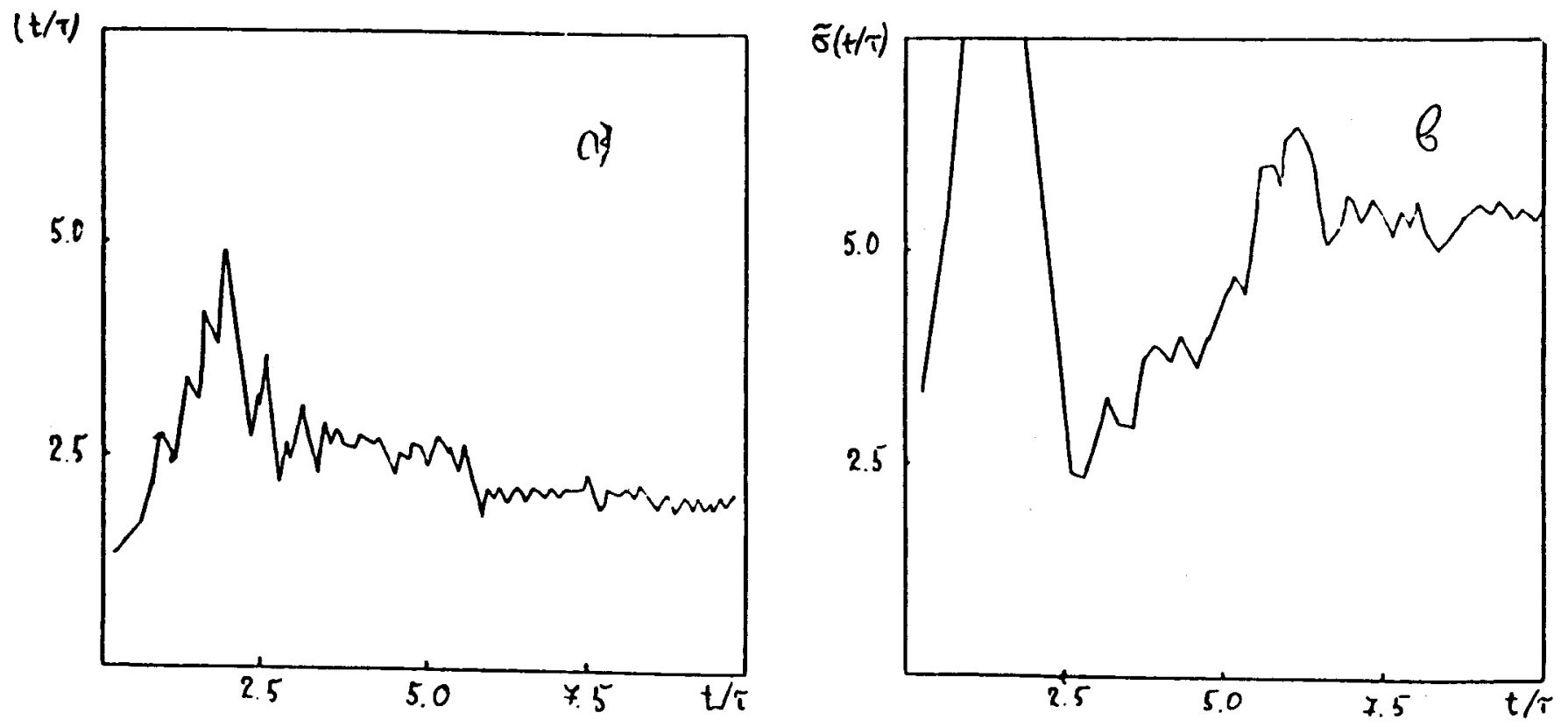

Figure 5: The raximum Liapunov exponent $\sigma_{1}(t)$ in the case without (a) and (b)
significant dark matter. 


\section{REFERENCES}

[1] Aarseth, S.J. (1963), MNKA S, 126, 223.

[2] Agekian, T.A.,Ano sova, J.P.(1977), Trudy Astron.obs. Leningr. Univ., 32,115 .

[3] Alladin, S.M. (1988), in M.J.Valtonen (ed.), The Few Body Problem, D.Keidel Publ .Co., Dordrecht, Holland, 216.

[4] A mo sova, J.P.(1986), A strophys. Space Sci.,124, 217.

[5] A no sova, J.P., Zavalov, N.N.(1988), Sov. A stron.Zh.63, 283.

[6] Butten, A. (1975), Double and Multiple Stars, Springer Verlag, New York.

[7] Casertel11,L. et.al., (1976), Phys. Rev.A., 13,1276.

[8] Gorbatsky, V.G. (1986), Introduction into Physics of Galaxies and Galaxy Clusters, Nauka, Mo scow.

[9] Karachent seva,V.E. et al.(1979), Izv. SAO, 7,3.

[10] Karachetseva, V.E. et al.(1987), Izv. SAO, 15, 21.

[11] Lichtenberg, A.J., Lieberman, M.A.(1983), Regular and Stochastic Motions, Springer Verlag, New York.

[12] Mamo n, G. (1987), Astrophys.J ., $298,527$.

[13] Roos, N., Norman,G.A. (1979), A stron. Astrophys., 76, 75.

[14] Szebehely, V. (1973), Recent Adv. in Astron., 5, 23.

15 Val tonen, M.(1988), Vistas Astron., 32,23. 